\title{
A First Comparison of Millimeter Continuum and Mg II Ultraviolet Line Emission from the Solar Chromosphere
}

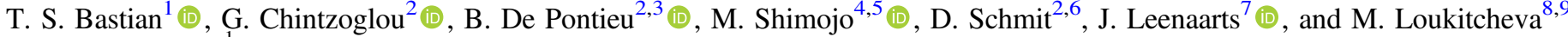 \\ National Radio Astronomy Observatory, 520 Edgemont Road, Charlottesville, VA 22903, USA; tbastian@nrao.edu \\ ${ }^{2}$ Lockheed Martin Solar \& Astrophysics Lab, Org. A021S, Building 252, 3251 Hanover Street, Palo Alto, CA 94304, USA \\ Institute of Theoretical Astrophysics, University of Oslo, P.O. Box 1029 Blindern, NO-0315 Oslo, Norway \\ ${ }^{4}$ National Astronomical Observatory of Japan, 2-21-1, Osawa, Mitaka, Tokyo 181-8588, Japan \\ ${ }^{5}$ Department of Astronomical Science, The Graduate University for Advanced Studies (SOKENDAI), 2-21-1, Osawa, Mitaka, Tokyo 181-8588, Japan \\ ${ }^{6}$ Bay Area Environmental Research Institute, 625 2nd Street, Suite 209, Petaluma, CA 94952, USA \\ ${ }^{7}$ Institute for Solar Physics, Department of Astronomy, Stockholm University, AlbaNova University Centre, SE-106 91 Stockholm, Sweden \\ ${ }^{8}$ Center For Solar-Terrestrial Research, New Jersey Institute of Technology, 323 Martin Luther King Boulevard, Newark, NJ 07102, USA \\ ${ }^{9}$ Max-Planck-Institut für Sonnensystemforschung, Justus-von-Liebig-Weg 3, D-37077 Göttingen, Germany \\ Received 2017 June 15; revised 2017 August 3; accepted 2017 August 3; published 2017 August 18
}

\begin{abstract}
We present joint observations of the Sun by the Atacama Large Millimeter/submillimeter Array (ALMA) and the Interface Region Imaging Spectrograph (IRIS). Both millimeter/submillimeter- $\lambda$ continuum emission and ultraviolet (UV) line emission originate from the solar chromosphere and both have the potential to serve as powerful and complementary diagnostics of physical conditions in this enigmatic region of the solar atmosphere. The observations were made of a solar active region on 2015 December 18 as part of the ALMA science verification effort. A map of the Sun's continuum emission was obtained by ALMA at a wavelength of $1.25 \mathrm{~mm}$ (239 GHz). A contemporaneous map was obtained by IRIS in the Mg II h doublet line at $2803.5 \AA$. While a clear correlation between the $1.25 \mathrm{~mm}$ brightness temperature $T_{B}$ and the $\mathrm{Mg} \mathrm{II} \mathrm{h}$ line radiation temperature $T_{\text {rad }}$ is observed, the slope is $<1$, perhaps as a result of the fact that these diagnostics are sensitive to different parts of the chromosphere and that the $\mathrm{Mg}$ II $\mathrm{h}$ line source function includes a scattering component. There is a significant difference $(35 \%)$ between the mean $T_{B}(1.25 \mathrm{~mm})$ and mean $T_{\text {rad }}(\mathrm{Mg}$ II). Partitioning the maps into "sunspot," "quiet areas," and "plage regions" we find the relation between the IRIS Mg II h line $T_{\text {rad }}$ and the ALMA $T_{B}$ regiondependent. We suggest this may be the result of regional dependences of the formation heights of the IRIS and ALMA diagnostics and/or the increased degree of coupling between the UV source function and the local gas temperature in the hotter, denser gas in plage regions.
\end{abstract}

Key words: Sun: chromosphere - Sun: radio radiation - Sun: UV radiation

\section{Introduction}

The solar chromosphere is a region of the solar atmosphere, immediately above the radiation-dominated photosphere, in which non-radiative heating first becomes manifest. It is a highly dynamic layer through which acoustic waves propagate from below and shock, dissipating their energy. The ionization fraction varies by orders of magnitude within the chromosphere, while the plasma beta (ratio of gas to magnetic pressures) transitions from generally greater than unity in the underlying photosphere (plasma control) to generally less than unity in the overlying corona (magnetic control), leading to an interface region of significant magnetic complexity. Hence, establishing the structure and dynamics of the solar chromosphere and understanding the transfer of radiative and mechanical energy through the chromosphere pose significant challenges.

Optical and UV line emission, as well as infrared, millimeter, and submillimeter wavelength continuum emission, originate from the chromosphere and have the potential to serve as powerful probes of its structure and dynamics, but their utility has sometimes been limited by either their accessibility to observation (UV) or by technical challenges (millimeter/ submillimeter- $\lambda$ ). Recently, high spatial/spectral resolution observations of the solar chromosphere in the UV became possible thanks to the Interface Region Imaging Spectrograph (IRIS) small explorer space mission (De Pontieu et al. 2014).
Among the newly accessible UV diagnostics, the $\mathrm{Mg}$ II $\mathrm{h}$ and $\mathrm{k}$ doublet lines near $2800 \AA$ are of particular interest. Magnesium is $\sim 15 \times$ more abundant than calcium in the solar atmosphere; the $\mathrm{Mg} \mathrm{h}$ and $\mathrm{k}$ lines therefore sample a wider range of heights of the solar chromosphere than the traditionally used optical $\mathrm{Ca}$ II $\mathrm{H}$ and $\mathrm{K}$ lines (near $3950 \AA$ ). Moreover, the Mg II line formation is simpler than that of other commonly used diagnostics such as the $\mathrm{He}$ I $10830 \AA$ line and $\mathrm{H} \alpha$ (Leenaarts et al. 2013a). Interest in the $\mathrm{Mg} \mathrm{II} \mathrm{h}$ and $\mathrm{k}$ lines as a means of diagnosing key parameters at chromospheric heights has therefore been strong and effort has been focused on modeling their radiative transfer in the context of chromospheric models (Leenaarts et al. 2013a, 2013b; Pereira et al. 2013; Sukhorukov \& Leenaarts 2017).

Continuum observations at millimeter and submillimeter wavelengths are also an attractive probe of the chromosphere (e.g., Bastian 2002). The dominant sources of opacitycollisions between free electrons and ions (free-free opacity) and between free electrons and neutral hydrogen $\left(\mathrm{H}^{-}\right.$opacity) - are well understood; the radiation is formed under conditions of LTE and the source function is therefore Planckian. Since $h \nu / K T \ll 1$ the Rayleigh-Jeans approximation is valid and the observed intensity or, equivalently, brightness temperature $T_{B}$ is linearly proportional to the temperature of the emitting material, $T_{\text {gas }}$. We note, however, that the degree of ionization for hydrogen and helium can depart significantly from the expectations of statistical ionization equilibrium in the solar 
chromosphere (Carlsson \& Stein 2002; Leenaarts et al. 2007; Golding et al. 2016); thus, while the source function is in LTE, the opacity can be far from its LTE value (Wedemeyer-Böhm et al. 2007). The primary disadvantage to observations at millimeter/submillimeter- $\lambda$ has been the relatively poor angular resolution available in past years as a result of the fact that most observations were acquired using single dishes (e.g., White et al. 2017).

With the advent of the the Atacama Large Millimeter/ submillimeter Array (ALMA), high-resolution observations of the Sun are now possible and direct comparisons with other diagnostics of the solar atmosphere have become feasible. In this Letter, we present a first comparison of solar observations of the chromosphere in a solar active region made by ALMA in the $1.25 \mathrm{~mm}$ band $(239 \mathrm{GHz})$ with those of the same region made in the ultraviolet $\mathrm{Mg}$ II $\mathrm{h}$ doublet line by IRIS. It is convenient to compare the millimeter- $\lambda$ continuum brightness temperature $T_{B}$ with the analogous quantity, $T_{\text {rad }}$, the $\mathrm{Mg} \mathrm{II} \mathrm{h}$ line radiation temperature. Converting the millimeter- $\lambda$ continuum and $\mathrm{Mg}$ II line intensities to $T_{B}$ and $T_{\text {rad }}$ is a way of taking out the wavelength dependence of the Planck function, and so allows a meaningful comparison between the two. Leenaarts et al. (2013b) show, based on quiet-Sun-like models, that there is a relation between $\mathrm{h} 2 / \mathrm{k} 2$ peak radiation temperature and the gas temperature at the formation height of the peak (see their Figure 6). The gas temperature is roughly 500-1000 K higher than the radiation temperature because of the partial decoupling of the source function and the Planck function. The observations presented here represent the first opportunity to make a direct comparison of millimeter- $\lambda$ and UV line emission from the solar chromosphere, a preliminary benchmark against which to assess model calculations. In Section 2, we briefly describe the instruments and the observations. In Section 3, we present maps of the $1.25 \mathrm{~mm}$ continuum brightness temperature and the UV $\mathrm{Mg}$ II $\mathrm{h}$ line radiation temperature. We discuss millimeter/UV-wavelength correlations for various regions and suggest underlying reasons for their attributes. We conclude in Section 4.

\section{Observations and Analysis}

\subsection{Atacama Large Millimeter/submillimeter Array}

ALMA is a powerful, general purpose interferometer comprised of the $12 \mathrm{~m}$ array $(50 \times 12 \mathrm{~m}$ antennas $)$ and the Atacama Compact Array $(12 \times 7 \mathrm{~m}$ antennas and $4 \times 12 \mathrm{~m}$ total power antennas). ALMA is designed to perform imaging observations and spectroscopy of astrophysical phenomena in frequency bands that will ultimately span $35-950 \mathrm{GHz}$ or wavelengths of $0.32-8.6 \mathrm{~mm}$. Limited solar observing modes were made available to the scientific community in 2016 (ALMA Cycle 4): specifically, continuum observations are possible in band $3(100 \mathrm{GHz} / 3 \mathrm{~mm})$ and band $6(239 \mathrm{GHz} /$ $1.25 \mathrm{~mm})$. In order to observe the Sun with ALMA, special hardware modifications and calibration procedures were developed. The testing and commissioning effort that enabled solar observing with ALMA in bands 3 and 6 is described in detail by Shimojo et al. (2017) and White et al. (2017).

The final solar testing campaign prior to the release of solar observing modes in ALMA Cycle 4 was conducted in 2015 December. A variety of targets and modes were observed to ensure that systems performed as expected. Among the observations were band 6 observations of NOAA active region number 12470 on 2015 December 18 from 19:11:41 to 20:09:59 UT when the active region was at approximately N15E05. The band 6 observations were acquired in four spectral windows, each $2 \mathrm{GHz}$ in bandwidth, centered on 230 , 232, 246, and $248 \mathrm{GHz}$. The mean frequency was $239 \mathrm{GHz}$, or a wavelength of $1.25 \mathrm{~mm}$. The instantaneous field of view (FOV) of ALMA is determined by the primary beam of a single antenna: for band 6 the FWHM of an ALMA $12 \mathrm{~m}$ antenna is $24^{\prime \prime}$. While this is sufficient for some types of observing programs, the restricted FOV hampers others. For programs that require mapping an area larger than the antenna FOV, the interferometer is pointed to a grid of locations relative to a reference location, a technique referred to as "mosaicking." In doing so, care must be taken to correct the individual mosaic pointings for the physical ephemeris of the Sun and the Sun's differential rotation. The ALMA band 6 map of AR 12470 presented here was formed from a mosaic of 149 pointings, each pointing of a duration of $6.048 \mathrm{~s}$.

In order to produce maps calibrated in absolute terms, units of flux density or brightness temperature, it is necessary to recover all angular scales present in the target. An interferometer acts as a high-pass spatial filter. A map produced by interferometry alone filters out angular scales larger than those measured by the minimum spacing between two antennas. ALMA was carefully designed to recover all relevant angular scales in most cases, but for sources that are complex and extended it is necessary to use both interferometric and total power (TP) measurements. The TP antennas enable maps of the Sun on the largest angular scales, from the angular resolution of a single $12 \mathrm{~m}$ antenna to the angular scale of the Sun itself $\left(\approx 1920^{\prime \prime}\right)$, allowing power on all spatial frequencies to be restored. Calibration of the TP measurements is described by White et al. (2017) where the brightness temperature of the quiet Sun at the center of the solar disk is scaled to $5900 \mathrm{~K}$ $(1.25 \mathrm{~mm})$. The interferometric and TP mapping data were combined using "feathering" techniques as described by Shimojo et al. (2017). The $1.25 \mathrm{~mm}$ map of AR 12470 produced on 2015 December 18 is $143^{\prime \prime} \times 139^{\prime \prime}$ in size and has an angular resolution of $2 . " 4 \times 0 . " 9$ (position angle $-76^{\circ} .7$ ). The photometric calibration of the resulting map is dominated by the total power mapping measurements. These are expected to be calibrated in absolute terms to $\approx 5 \%$ (see White et al. 2017).

\subsection{Interface Region Imaging Spectrograph}

IRIS (De Pontieu et al. 2014) conducted high spatial $\left(0\right.$ ! $^{\prime} 16$ pix $\left.^{-1}\right)$, temporal, and spectral resolution $(0.025 \AA /$ pixel $)$ observations of the $\mathrm{Mg}$ II h line at $2803.5 \AA$ of $\mathrm{AR} 12470$ in coordination with ALMA. The IRIS data presented here were obtained on 2015 December 18 starting at 19:33:13 UT. They are comprised of three dense (0."35) 320-step very large raster scans (FOV $\left.112^{\prime \prime} \times 175^{\prime \prime}\right)$ centered at $(x, y)=\left(-59^{\prime \prime} \times 231^{\prime \prime}\right)$. Spatial binning $(\times 2)$ was performed on the slit dimension and also on the spectra (again $\times 2$ ). The exposure time was $2 \mathrm{~s}$, and each raster scan was obtained in $1026 \mathrm{~s}$ (with a total duration for the observation of $3078 \mathrm{~s}$ ). We present the first large, dense raster-from 19:33:13 to 19:51:09 UT. All spectral data sets were corrected as appropriate for instrumental effects such as dark subtraction, flat-fielding, and geometric and wavelength corrections.

Although the $\mathrm{Mg}$ II $\mathrm{k}$ line is of somewhat greater intensity than the $\mathrm{h}$ line, its profile contains a Manganese line in the blue 
wing, thereby complicating profile fitting. We therefore limit our analysis here to the $\mathrm{Mg}$ II $\mathrm{h}$ line at $2803.5 \AA$. The average solar $\mathrm{Mg}$ II $\mathrm{h}$ line profile is an emission line with a reversed core (yielding a red " $\mathrm{h} 2 \mathrm{r}$ " and a blue " $\mathrm{h} 2 \mathrm{v}$ " peak on either side of the " $\mathrm{h} 3$ " core). However, pertinent to the local conditions, the opacity modulates the double-peaked profile, making it asymmetric or even single peaked. We used a two-component model (Schmit et al. 2015), based on MPFIT (Markwardt 2008), to match the line emission core with a linear combination of a wide positive-amplitude Gaussian and a narrower negative-amplitude Gaussian. The model fits were used to derive intensities and wavelengths for both the red and blue $\mathrm{Mg}$ II h2 peaks that were then stored in separate arrays. Wherever the line core was not self-reversed, the intensity and wavelength of the single peak were recorded.

We then converted the intensity rasters for the derived peak fits from DN s${ }^{-1}$ to physical intensity units (erg s ${ }^{-1} \mathrm{~cm}^{-2} \mathrm{sr}^{-1}$ $\AA^{-1}$ ) using radiometric calibration version " 004 ," with an uncertainty of $\approx 15 \%$. The absolute intensity was then converted to radiation temperature $T_{\mathrm{rad}}$ via the Planckian for each peak (h2r, h2v, or single h2) that we measured. For the purposes of comparing $\mathrm{Mg}$ II $\mathrm{h}$ line radiation temperatures with ALMA brightness temperatures, we used the average of $h 2 \mathrm{v}$ and h2r $T_{\text {rad }}$ or the single h2 $T_{\text {rad }}$.

\section{Results and Discussion}

In order to compare the IRIS raster data with ALMA imaging data several steps were taken. The ALMA map was obtained in the geocentric coordinate frame (R.A. and decl.). It was therefore necessary to correct the final map for the solar position angle. For IRIS, pixels for which the fitting procedure failed $(<0.1 \%)$ were filled through the use of a median filter. There was a small amount of position "offset" from raster to raster. To correct for this, the IRIS raster data were interpolated onto a uniform grid $(0$ " $4 \times 0$ " 4 pixels $)$. Then, the resulting maps of $T_{\text {rad }}$ in the $\mathrm{Mg}$ II h2r, h2v, or single h2 line was convolved with a 2 ".9 $\times 0$ ".9 Gaussian kernel with the appropriate position angle so that it had the same angular resolution as the corresponding ALMA map. We note that the conversion of the $\mathrm{Mg}$ II $\mathrm{h}$ line intensity to radiation temperature removes the nonlinearity of the Planck function in the UV so that the subsequent smoothing step does not bias the radiation temperature, as would have been the case had the steps had been reversed. Finally, the maps were corrected for position offsets and differing FOVs to bring them into alignment, to allow comparisons to be made on a pixel by pixel basis. Note, however, that we have not corrected for the fact that pixels are correlated on the scale of ALMA's resolution, i.e., the pixel size is 0 " $4 \times 0$ ". 4 and they are correlated on an angular scale of 2 "! $4 \times 0$ " 9 .

The matched-resolution maps obtained by ALMA and IRIS are shown in Figure 1. A clear correlation is evident between the ALMA map of the $1.25 \mathrm{~mm}$ continuum brightness temperature $T_{B}$ and the IRIS map of the $\mathrm{Mg}$ II $\mathrm{h}$ line radiation temperature $T_{\text {rad }}$. To better characterize the nature of the correlation we formed scatter plots of the ALMA and IRIS observations as shown in Figure 2. The central panel shows a scatter plot of all pixels in common with the two maps (gray pixels). While the trend remains clear, the scatter of pixels is significant. The Pearson correlation coefficient for all pixels is 0.80 . We note that the mean and range of $1.25 \mathrm{~mm}$ continuum brightness temperatures are approximately $7380 \mathrm{~K}$ and
$5200-8700 \mathrm{~K}$, respectively, whereas the mean and range of the $\mathrm{Mg}$ II $\mathrm{h}$ radiation temperatures are approximately $5440 \mathrm{~K}$ and $4500-6500 \mathrm{~K}$, respectively. Hence, there is a significant offset between $T_{B}(1.25 \mathrm{~mm})$ and $T_{\text {rad }}(\mathrm{Mg}$ II) and a compression in range of the latter relative to the former. A linear fit to the data yields a slope of $m=0.55$. The causes for the slope, the offset, and the degree of scatter between the $1.25 \mathrm{~mm}$ brightness temperature and the radiation temperature derived from $\mathrm{Mg}$ II $\mathrm{h}$ are not fully clear. Preliminary results from advanced numerical models (e.g., those computed with the Bifrost model; Gudiksen et al. 2011 see also Carlsson et al. 2016 and references therein) of quiet-Sun conditions indicate that the Mg II h2v feature forms in the middle chromosphere over a range of heights from 600 to $2000 \mathrm{~km}$ with a peak around $1400 \mathrm{~km}$ (Leenaarts et al. 2013b). Synthetic observables calculated from the Bifrost at a wavelength of $1.3 \mathrm{~mm}$, near that reported here, suggest that the radiation mostly originates at similar heights, with a peak at $1150 \mathrm{~km}$ (Loukitcheva et al. 2017). While the diagnostics are formed in roughly the same region of the atmosphere, it is unclear whether there are systematic differences in formation height along individual lines of sight. For both diagnostics the formation height surface is highly corrugated with large variability expected from location to location. It is possible that the height difference between these diagnostics is larger than predicted by the models. There are already indications that the Bifrost model does not properly reproduce the middle to upper chromosphere where significant opacity for both IRIS and ALMA diagnostics occurs, as exemplified by the large discrepancy in line width between observed and synthetic $\mathrm{Mg}$ II $\mathrm{h}$ profiles from the simulations (Leenaarts et al. 2013a). In particular, the Bifrost model appears to lack opacity from upper chromospheric features such as spicules and fibrils. Hence, one possibility is that the observed slope is in part caused by a differing sensitivity of the diagnostics to local conditions in such features, with ALMA more sensitive and IRIS less sensitive because of the scattering nature of the $\mathrm{Mg}$ II line. It is also possible that the compressed temperature range deduced from the optically thick Mg II h line feature could be caused by radiative transfer effects. Since the $\mathrm{Mg}$ II $\mathrm{h}$ and $\mathrm{k}$ lines are scattering lines, the source function has a scattering contribution that leads to a source function that is not coupled to the local conditions and is lower than the local Planck function. Leenaarts et al. (2013b) also show that while the model correlation is good between the $\mathrm{Mg}$ II line radiation temperature and gas temperature, the radiation temperature underestimates the gas temperature by $\sim 500 \mathrm{~K}$ (much smaller than the offset between $T_{\text {rad }}\left(\mathrm{Mg}\right.$ II) and $T_{B}(1.25 \mathrm{~mm})$ observed). However, at low intensities the correlation shows a significant scatter with many pixels having gas temperatures that are significantly higher than $T_{\text {rad }}$ (Mg II) - again the result of radiation scattering. While the slope and scatter of the correlation may be qualitatively explained, in part, the significant offset between $T_{\text {rad }}\left(\mathrm{Mg}\right.$ II) and $T_{B}(1.25 \mathrm{~mm})$ is not understood within the context of current modeling efforts. We caution that it is in any case unclear how applicable these quiet-Sun models are to our observations of AR 12470, where even the quietest regions are affected by the super-penumbra that surrounds the sunspot and plage. Nevertheless, taken together these observations illustrate how millimeter- $\lambda$ and UV line observations may be used to impose stringent constraints on future numerical models of the chromosphere. 

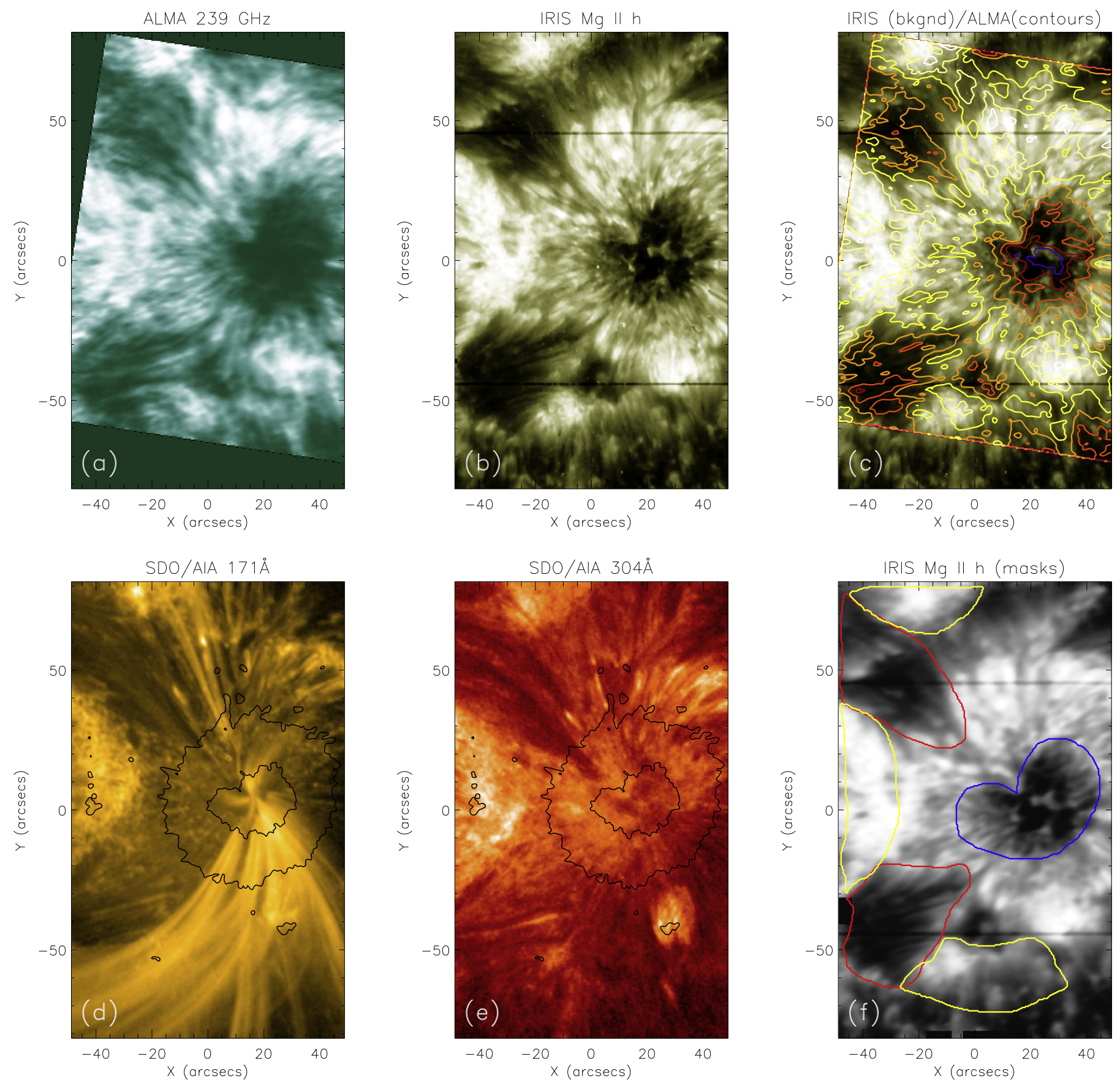

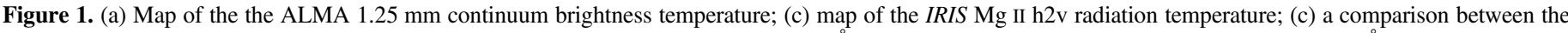

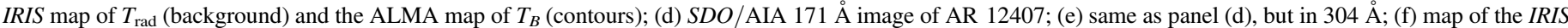

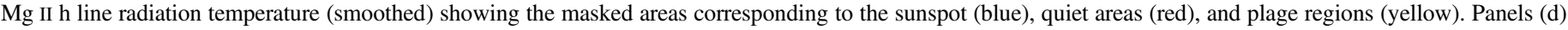
and (e) also show contours of the sunspot umbra and penumbra based on the SDO Heliospheric Magnetic Imager white light continuum image.

It is informative to subdivide the ALMA and IRIS maps. To do so, portions of AR 12470 covered by both instruments were identified as "sunspot," "quiet" areas (see comment above about the impact of the super-penumbra), and plage regions. Figure 2(f) shows the corresponding spatial masks as blue, red, and yellow contours, respectively. Considering each of these separately shows that the correlation between the $1.25 \mathrm{~mm}$ brightness temperature and the $\mathrm{Mg}$ II $\mathrm{h}$ line radiation temperature is region-dependent. The solid line in Figure 2 shows a linear fit to sunspot pixels (slope $m=0.39$ ); the dashed line shows the same for "quiet" regions pixels $(m=0.55)$, and the dotted-dashed line shows the same for plage regions $(m=0.68)$. The reason for this behavior is not fully understood. In an analysis of IRIS Mg II $\mathrm{k}$ line profiles in plage regions, Carlsson et al. (2015) suggest that the observed line profiles arise naturally in hot, dense plasma, ensuring better coupling of the source function to the local temperature. Hence, the increased slope observed in the correlation between the $1.25 \mathrm{~mm}$ continuum emission and the $\mathrm{Mg}$ II $\mathrm{h}$ line may similarly reflect a higher degree of coupling of the source function to the local gas temperature.

We have checked sources of possible systematic error in both data sets to ensure that the attributes we have described are robust. Chief among these is the absolute calibration of the two 


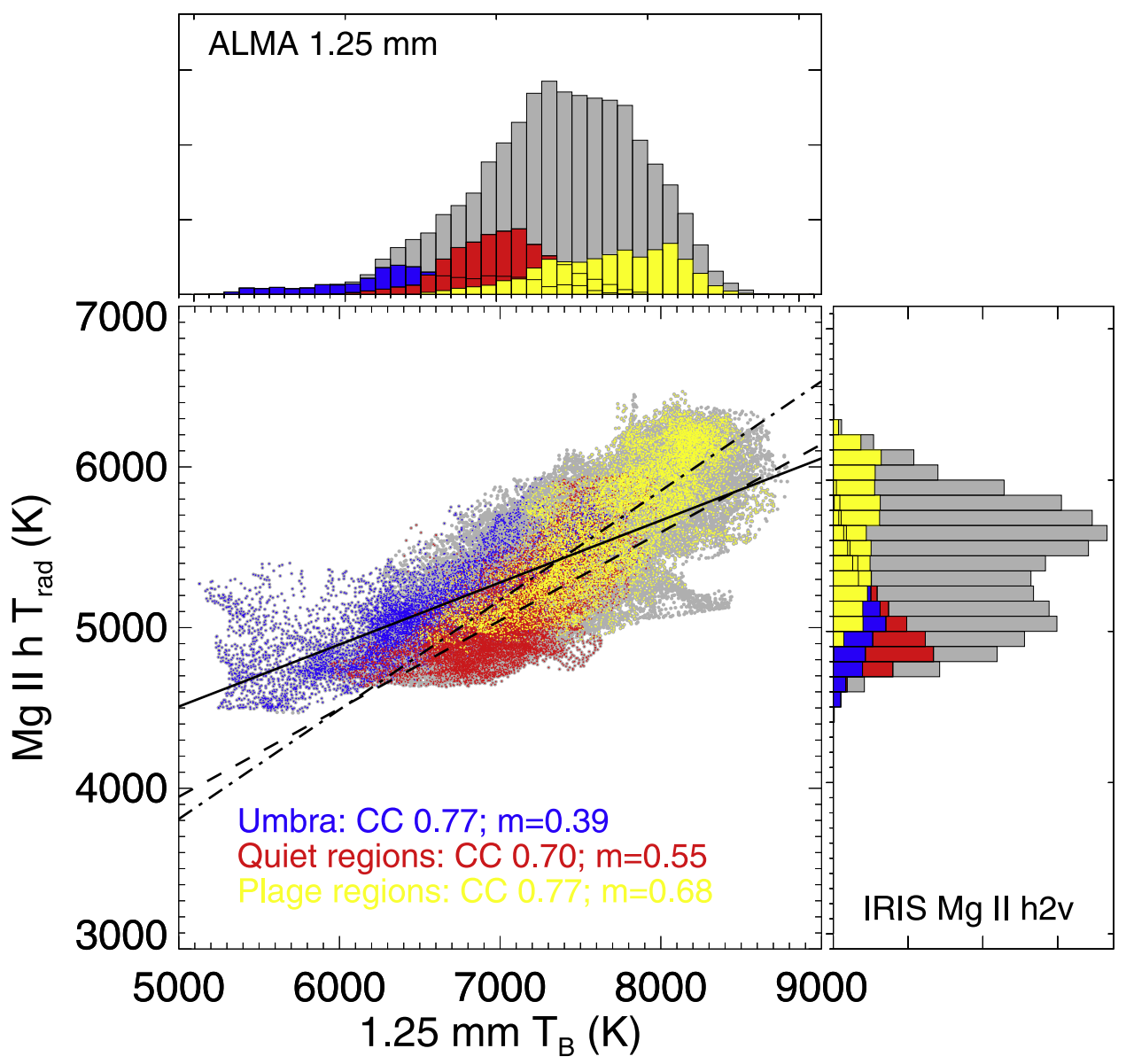

Figure 2. Main panel shows a scatter plot of IRIS Mg II h line radiation temperatures vs. the corresponding ALMA 1.25 mm continuum brightness temperatures. Gray points represent all pixels in common with the two maps. Blue points mark pixels in the sunspot umbra, red for "quiet" areas, and yellow for "plage" regions. The corresponding histograms are shown for the ALMA data (top panel) and the IRIS data (right panel). The mean 1.25 mm brightness temperature of the sunspot, quiet, and plages are $6555 \mathrm{~K}, 7045 \mathrm{~K}$, and $7735 \mathrm{~K}$, whereas the mean $\mathrm{Mg}$ II h line radiation temperatures are $5109 \mathrm{~K}, 5069 \mathrm{~K}$, and $5672 \mathrm{~K}$, respectively. Linear fits to pixels marking umbra, quiet, and plage pixels are shown as a solid, dashed, and dotted-dashed line, respectively.

data sets. As noted in previous sections, the ALMA brightness scale is dominated by the total power measurements, believed to be accurate to $\approx 5 \%$, which then carries over directly to the brightness temperature uncertainty. Radiometric calibration of the IRIS observations is of order $15 \%$ which, although larger than that of ALMA, implies a smaller radiation temperature uncertainty $(\lesssim 2 \%)$ owing to the exponential of the Planck function. Another possible source of error is the fact that the two data sets were not obtained strictly simultaneously-the IRIS data were obtained during the course of $\sim 18$ minutes during ALMA mosaic mapping ( $\sim 50$ minute duration). While time variability likely increases the scatter in the correlation between the two data sets, it cannot account for the offsets and slopes of the scatter plots. In the case of IRIS, using contemporaneous sparse raster data, we estimate an rms time variation of $\sigma=300 \mathrm{~K}$ in the sunspot umbral region (largely due to oscillations) and $\sigma=200 \mathrm{~K}$ in plage regions. ALMA data at $1.25 \mathrm{~mm}$ suitable for characterizing the time variability of the continuum $\mathrm{mm}$ emission are not available for this active region. A study of the $\mathrm{mm}$ and UV time variability in sunspot, quiet, and plage regions lies outside the scope of this work and will be pursued separately. Another consideration in the case of ALMA is whether the contribution of the overlying corona to the observed brightness temperature, $\Delta T_{B}(1.25 \mathrm{~mm})$, is significant. The dominant source of opacity over the active region at millimeter- $\lambda$ is free-free absorption. The optical depth of a differential layer $d z$ overlying the active region is $d \tau \approx 0.2(\lambda / c)^{2} n_{e}^{2} T^{-3 / 2} d z$ (Dulk 1985). The brightness temperature contribution of the overlying (optically thin) coronal material at $1.25 \mathrm{~mm}$ can then be estimated as

$$
\begin{aligned}
\Delta T_{B}(1.25 \mathrm{~mm}) & \approx \int T d \tau \approx 0.2 \frac{\lambda^{2}}{c^{2}} \int T^{-1 / 2} n_{e}^{2} d z \\
& =0.2 \frac{\lambda^{2}}{c^{2}} \int T^{-1 / 2} \phi(T) d T,
\end{aligned}
$$

where $\phi(T)=n_{e}^{2}(T) d z / d T$ is the differential emission measure of the coronal material over AR 12470. We estimated $\phi(T)$ using measurements by the Solar Dynamics Observatory Atmospheric Imaging Assembly and found that $\Delta T_{B}(1.25 \mathrm{~mm})$ was no more than $2 \%$. We conclude that none of the potential sources of systematic error likely has a significant impact on the ALMA/ IRIS correlation properties observed.

\section{Summary and Conclusions}

We have presented a first comparison of high angular resolution observations of the $1.25 \mathrm{~mm}$ continuum emission from a solar active region (AR 12470) made by ALMA on 
2015 December 18 with nearly simultaneous observations of the Mg II h line obtained by IRIS. The two radiation regimes hold promise as powerful and complementary diagnostics of the structure and dynamics of the solar chromosphere. In comparing maps of the ALMA $1.25 \mathrm{~mm}$ brightness temperature and the IRIS $\mathrm{Mg}$ II $\mathrm{h}$ radiation temperature we find a clear correlation between $T_{B}(1.25 \mathrm{~mm})$ and $T_{\text {rad }}(\mathrm{Mg}$ II) although the $\mathrm{Mg}$ II radiation temperatures are offset to significantly lower values than the corresponding $1.25 \mathrm{~mm}$ brightness temperatures. Furthermore, the slope of the correlation is significantly smaller than unity. While the temperature offset is not understood, the slope $<1$ may, in part, be the result of the fact that the $\mathrm{Mg}$ II $\mathrm{h}$ line source function has a radiation scattering component, also compatible with the the significant scatter in the correlation. Sub-dividing the active region maps into sunspot umbra, quiet areas, and plage regions, we find that the correlation between $T_{B}(1.25 \mathrm{~mm})$ and $T_{\mathrm{rad}}(\mathrm{Mg}$ II) depends on the sub-region. The slopes of the linear fits are $0.39,0.55$, and 0.68 , respectively. Qualitatively, the larger slope measured in plage regions may be due to a higher degree of coupling of the source function to the gas under the hotter, denser conditions expected in those regions. These observations highlight the need for chromospheric models not only for quiet-Sun conditions, but of active region conditions as well. Future work will focus on expanding millimeter/submillimeter- $\lambda$ continuum and UV line joint observations to a variety of chromospheric environments and comparing them with the numerical models in detail as a means of better utilizing the two disparate wavelength regimes as complementary probes of the chromosphere.

The National Radio Astronomy Observatory is a facility of the National Science Foundation operated under cooperative agreement by Associated Universities, Inc. This Letter makes use of the following ALMA data: ADS/JAO.ALMA \#2011.0.00020.SV. ALMA is a partnership of ESO (representing its member states), NSF (USA) and NINS (Japan), together with NRC (Canada), NSC and ASIAA (Taiwan), and KASI (Republic of Korea), in cooperation with the Republic of Chile. The Joint ALMA Observatory is operated by ESO, AUI/NRAO and NAOJ. IRIS is a NASA small explorer mission developed and operated by LMSAL with mission operations executed at NASA Ames Research center and major contributions to downlink communications funded by ESA and the Norwegian Space Centre. M.S. was supported by JSPS
KAKENHI grant number JP17K05397. This work was partly carried out on the solar data analysis system and common-use data analysis computer system operated by ADC/ NAOJ. G.C. and B.D.P. are both supported by NASA contract NNG09FA40C (IRIS). M.L. acknowledges NSF grant AST1312802 and NASA grant NNX14AK66G. J.L. is supported by the CHROMOBS and CHROMATIC grants of the Knut and Alice Wallenberg foundation. SDO data was made available courtesy of NASA/SDO and the AIA, EVE, and HMI science teams.

Facilities: ALMA, IRIS, SDO.

\section{ORCID iDs}

T. S. Bastian (1) https://orcid.org/0000-0002-0713-0604

G. Chintzoglou (i) https://orcid.org/0000-0002-1253-8882

B. De Pontieu (i) https://orcid.org/0000-0002-8370-952X

M. Shimojo iㅏ https://orcid.org/0000-0002-2350-3749

J. Leenaarts (1) https://orcid.org/0000-0003-4936-4211

\section{References}

Bastian, T. S. 2002, AN, 323, 271

Carlsson, M., Hansteen, V. H., Gudiksen, B. V., Leenaarts, J., \& De Pontieu, B. 2016, A\&A, 585, A4

Carlsson, M., Leenaarts, J., \& De Pontieu, B. 2015, ApJL, 809, L30

Carlsson, M., \& Stein, R. F. 2002, ApJ, 572, 626

De Pontieu, B., Title, A. M., Lemen, J. R., et al. 2014, SoPh, 289, 2733

Dulk, G. A. 1985, ARA\&A, 23, 169

Golding, T. P., Leenaarts, J., \& Carlsson, M. 2016, ApJ, 817, 125

Gudiksen, B. V., Carlsson, M., Hansteen, V. H., et al. 2011, A\&A, 531, A154

Leenaarts, J., Carlsson, M., Hansteen, V., \& Rutten, R. J. 2007, A\&A, 473, 625

Leenaarts, J., Pereira, T. M. D., Carlsson, M., Uitenbroek, H., \& De Pontieu, B. 2013a, ApJ, 772, 90

Leenaarts, J., Pereira, T. M. D., Carlsson, M., Uitenbroek, H., \& De Pontieu, B. 2013b, ApJ, 772, 89

Loukitcheva, M., White, S. M., Solanki, S. K., Fleishman, G. D., \& Carlsson, M. 2017, A\&A, 601, A43

Markwardt, C. B. 2008, in Astronomical Data Analysis Software and Systems XVIII, ed. D. Bohlender, P. Dowler, \& D. Durand (San Francisco, CA: ASP), 251

Pereira, T. M. D., Leenaarts, J., De Pontieu, B., Carlsson, M., \& Uitenbroek, H. 2013, ApJ, 778, 143

Schmit, D., Bryans, P., De Pontieu, B., et al. 2015, ApJ, 811, 127

Shimojo, M., Bastian, T. S., Hales, A. S., et al. 2017, SoPh, 292, 87

Sukhorukov, A. V., \& Leenaarts, J. 2017, A\&A, 597, A46

Wedemeyer-Böhm, S., Ludwig, H. G., Steffen, M., Leenaarts, J., \& Freytag, B. 2007, A\&A, 471, 977

White, S. M., Iwai, K., Phillips, N. M., et al. 2017, SoPh, 292, 88 